\title{
Defining Bidimensional Fuzzy Sets for Visual Coarseness Modelling
}

\author{
J. Chamorro-Martínez, P. Martínez-Jiménez, and J.M. Soto-Hidalgo
}

\begin{abstract}
In this paper, the texture feature "coarseness" is modelled by means of fuzzy sets, relating representative coarseness measures (our reference set) with the human perception of this type of feature. In our study, a wide variety of measures have been analyzed, defining unidemsional and bidimensional fuzzy set for different combination of measures. The fineness human perception has been collected from polls filled by human subjects, performing an aggregation of their assessments by means of OWA operators. Using as reference set a combination of some measures, the membership function corresponding to the fuzzy set is modelled as the function which provides the best fit of the collected data.
\end{abstract}

\section{INTRODUCTION}

Texture is, together with the color and shape, one of the most important features used to analyze natural images. It is usual for humans to describe visual textures according to some vague "textural concepts" like coarseness/fineness, orientation or regularity [2], [17], [22]. From all of them, the coarseness/fineness is the most popular one, being common to associate the presence of fineness with the presence of texture (from this point of view, texture is defined as local variations against the idea of homogeneity). In this sense, a fine texture is considered as small texture primitives with big gray tone differences between neighbor primitives (e.g. the image in figure 1(A)), whereas a coarse texture corresponds to bigger primitives formed by several pixels (e.g. the image in figure 1(I)). By considering the importance of this textural concept, in this paper we will focus our study on the "fineness" modelling (let us remark that "coarseness" and "fineness" are opposite but related textural concepts).

There are many measures in the literature that, given an image, capture the fineness (or coarseness) presence in the sense that the greater the value given by the measure, the greater the perception of texture [9]. However, there is no perceptual relationship between the value given by these measures and the degree in which the humans perceive the texture. Thus, given a certain value calculated by applying a measure to an image, there is not an immediate way to decide whether there is a fine texture, a coarse texture or something intermediate (i.e. there is not a textural interpretation).

The imprecision associated to these fineness measures suggests the use of representation models that incorporate the

J. Chamorro-Martínez and P. Martínez-Jiménez are with the Department of Computer Science and Artificial Intelligence, University of Granada Spain. J.M. Soto-Hidalgo is with the Department of Computer Architecture, Electronics and Electronic Technology, University of Córdoba, Spain email:\{jesus,pedromartinez\}@decsai.ugr.es,jmsoto@uco.es

This work has been partially supported by the Spanish Government under the TEC2006-13845 and Consolider Ingenio 2010 MIPRCV (CSD200700018) projects. uncertainty. Nevertheless, the majority of the approaches that can be found in the literature are crisp proposals [5], [9], [18], [22] where uncertainty is not properly taken into account. To face this problem, fuzzy logic has been recently employed for representing the imprecision related to texture. However, in many of these approaches, fuzzy logic is usually applied just during the process but the output do not habitually model the imprecision (being often a crisp one). Examples of this fact are frequently found in the literature, like those approaches that use texture to perform image segmentation or classification on the basis of fuzzy clustering [8], [20], [23], fuzzy rules [6], [10], [16], etc.

Other interesting approaches emerge from the contentbased image retrieval scope, where semantic data is managed by means of fuzzy sets [12], [13]. In these proposals, a mapping from low-level statistical features to high level textural concepts is performed by defining membership functions for each textural feature. However, given a feature, these membership functions are not obtained by considering the relationship between the computational feature and the human perception of texture, so the linguistic labels related to these membership functions do not necessarily match what a human would expect.

In this paper we propose to solve this problem by representing the concept of fineness on the basis of fuzzy sets relating representative measures of the modelled texture (usually some statistic) with its human perception of fineness. In our analysis, a wide variety of measures have been considered, including classical statistical measures, frequency domain and fractal dimension measures, etc. Thus, this paper propose a group of fuzzy sets (defined on different measures) which model the textural fineness according to the human perception. The performance of each fuzzy set is analyzed and checked with the human assessments, proposing a subgroup of them as the most adequate for modelling fineness perception in texture images.

The rest of the paper is organized as follows. In section II a general overview introducing our methodology is presented. After that, some elements of the model are describe in detail in the following sections; concretely, the way to obtain human assessments about fineness-coarseness perception is faced in section III, while section IV describes the procedure employed to obtain the membership functions of the proposed fuzzy sets. In section $\mathrm{V}$ the results of applying the models are shown, and the main conclusions and future works are summarized in section VI. 

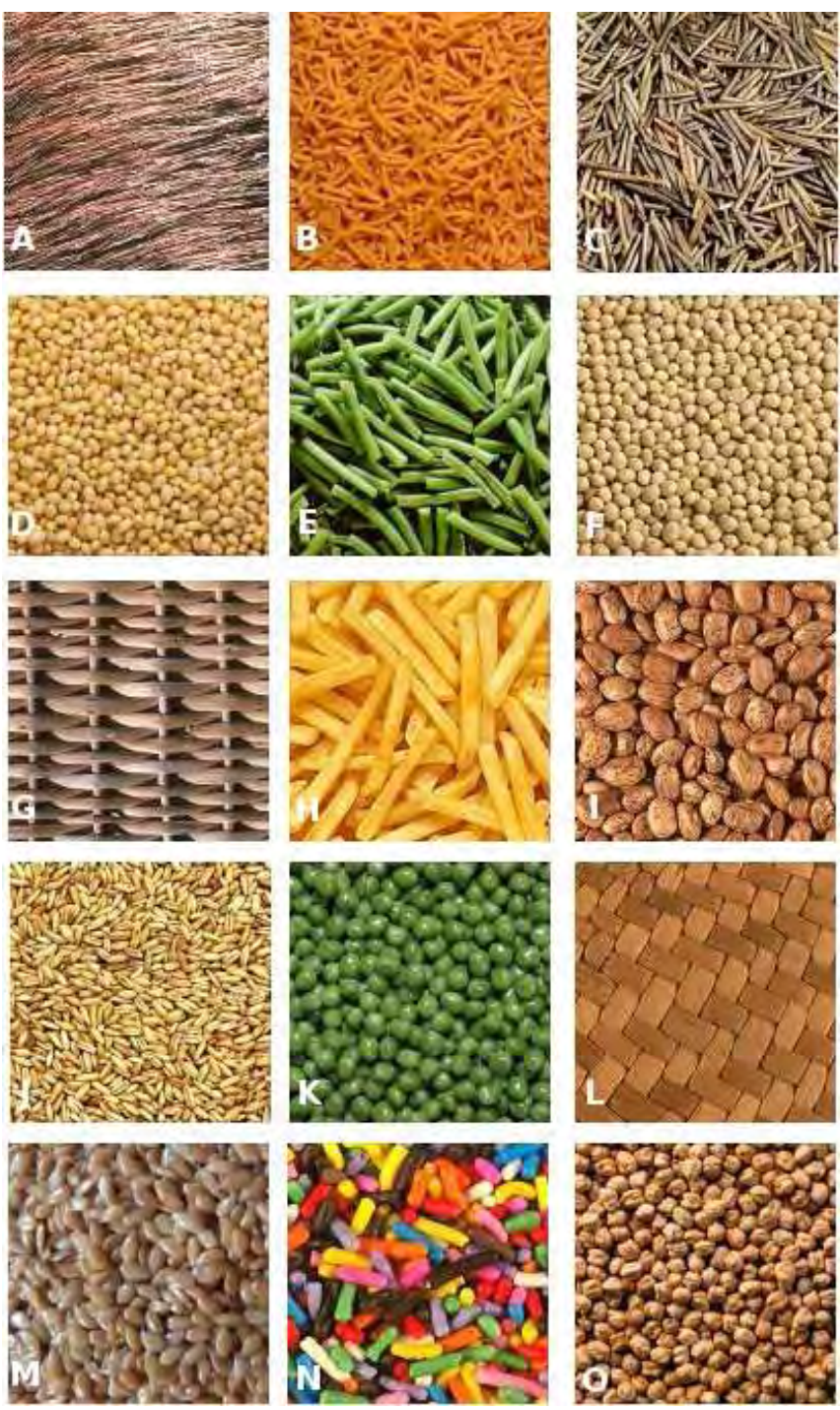

Fig. 1. Some examples of images with different degrees of fineness

\section{FuZzy Modelling of CoArseness: An Overview OF THE PROPOSAL}

In this paper we propose to model the coarseness-fineness perception as a fuzzy set. For this purpose, two elements need to be defined: (i) the domain of the reference set and (ii) the membership function.

Concern to the reference set, we will define the fuzzy set on the domain of a given set of coarseness-fineness measures. From now on, we will note $\mathcal{P}=\left\{P_{1}, \ldots, P_{K}\right\}$ the set of $K$ measures, $\mathcal{F}=\left\{F_{1}, \ldots, F_{K^{\prime}}\right\} \subseteq \mathcal{P}$ the subset of $K^{\prime} \leq$ $K$ measures selected from $\mathcal{P}$ to define the reference set ${ }^{1}$, and $\mathcal{T}_{\mathcal{F}}$ the fuzzy set defined on the domain of $\mathcal{F}$. In this paper, the set $\mathcal{P}=\left\{P_{1}, \ldots, P_{K}\right\}$ is formed by the $K=17$ measures shown in the first column of table I. It includes classical statistical measures, frequency domain approaches, fractal dimension analysis, etc. All of them are automatically

\footnotetext{
${ }^{1}$ Let us remark that given a set $\mathcal{P}$ of $K$ measures, the fuzzy set could be defined by using all the measures $\left(K^{\prime}=K\right)$ or a subset of them $\left(K^{\prime}<K\right)$
}

computed from the texture image.

With regard to the membership function, we propose to obtain it by learning a relationship between the fineness measures and the human perception of fineness. For this purpose, two questions will be faced: firstly, how to obtain the data about the "human perception of fineness" and, secondly, how to fit these data with the fineness measures in order to obtain the membership function.

To get information about human perception of fineness, a set of images covering different degrees of fineness will be gathered. This images will be used to collect, by means of a pool, human assessments about the perceived fineness. From now on, let $\mathcal{I}=\left\{I_{1}, \ldots, I_{N}\right\}$ be the set of $N$ images representing fineness-coarseness examples, and let $\Gamma=\left\{v^{1}, \ldots, v^{N}\right\}$ be the set of perceived fineness values associated to $\mathcal{I}$, with $v^{i}$ being the value representing the degree of fineness perceived by humans in the image $I_{i} \in \mathcal{I}$. The way to obtain $\Gamma$ will be describe in section III

To obtain the membership function, a robust fitting method will be applied in order to obtain suitable functions relating (i) the measures calculated for each image with (ii) the degree of fineness perceived by humans. To simplify the notation, as it is usual in the scope of fuzzy sets, we will use the same notation $\mathcal{T}_{\mathcal{F}}$ for the fuzzy set and for the membership function that defines it. The fitting stage will be describe in section IV .

\section{Assessment Collection}

In this section, the way to obtain the set $\Gamma=\left\{v 1, \ldots, v^{N}\right\}$ of perceived fineness values associated to $\mathcal{I}$ will be described. For this purpose, firstly the image set $\mathcal{I}$ will be selected. After that, a poll for getting assessments about the perception of fineness will be designed. Finally, for a given image, the assessments of the different subjects will be aggregated.

\section{A. The texture image set}

A set $\mathcal{I}=\left\{I_{1}, \ldots, I_{N}\right\}$ of $N=80$ images representative of the concept of fineness has been selected. Figure 1 shows some images extracted from the set $\mathcal{I}$. Such set has been selected satisfying the following properties:

1) It covers the different presence degrees of fineness.

2) The number of images for each presence degree is representative enough.

3) Each image shows, as far as possible, just one presence degree of fineness.

Due to the third property, each image can viewed as "homogeneous" respect to the fineness degree represented, i.e., if we select two random windows (with a dimension which does not "break" the original texture primitives and structure), the perceived fineness will be the same for each window (and also respect to the original image). In other words, we can see each image $I_{i} \in \mathcal{I}$ as a set of lower dimension images (windows) with the same fineness degree of the original one.

As we explained, given an image $I_{i} \in \mathcal{I}$, a set of measures $\mathcal{F}$ will be applied on it. In fact, and thanks to the 
third property, we really can apply these measures for each subimage, assuming that the human assessment associated to that subimage will be the human assessment associated to the whole image. From now on, we will note as $\mathbf{M}_{w}^{i}=$ $\left[m_{1}^{i, w}, \ldots, m_{K^{\prime}}^{i, w}\right]$ the vector of measures for the $w$-th window of the image $I_{i}$, with $m_{k}^{i, w}$ being the result of applying the measure $F_{k} \in \mathcal{F}$ to the $w$-th window of the image $I_{i}$.

\section{B. The poll}

Given the image set $\mathcal{I}$, the next step is to obtain assessments about the perception of fineness from a set of subjects. From now on we shall note as $\Theta^{i}=\left[o_{1}^{i}, \ldots, o_{L}^{i}\right]$ the vector of assessments obtained from $L$ subjects for the image $I_{i}$. To get $\Theta^{i}$, subjects will be asked to assign images to classes, so that each class has associated a perception degree of fineness.

In particular, 20 subjects have participated in the poll and 9 classes have been considered. The first nine images in figure 1 show the nine representative images for each class used in this poll. It should be noticed that the images are decreasingly ordered according to the presence degree of the fineness concept. The first class (Figure 1(A)) represents a presence degree of 1 while the ninth class (Figure 1(I)), represents a presence degree of 0 . The rest of the classes (Figure 1(B)-(H)) represent degrees in the interval $(0,1)$.

As result, a vector of 20 assessments $\Theta^{i}=\left[o_{1}^{i}, \ldots, o_{20}^{i}\right]$ is obtained for each image $I_{i} \in \mathcal{I}$. The degree $o_{j}^{i}$ associated to the assessment given by the subject $S_{j}$ to the image $I_{i}$ is computed as $o_{j}^{i}=(9-k) * 0.125$, where $k \in\{1, \ldots, 9\}$ is the index of the class $C_{k}$ to which the image is assigned by the subject.

\section{Assessment aggregation}

Our aim at this point is to obtain, for each image in the set $\mathcal{I}$, one assessment $v^{i}$ that summarizes the assessments $\Theta^{i}$ given by the different subjects about the presence degree of fineness.

To aggregate opinions we have used an OWA operator guided by a quantifier [25]. Concretely, the quantifier "the most" has been employed, which allows to represent the opinion of the majority of the polled subjects. This quantifier is defined as

$$
Q(r)=\left\{\begin{array}{lr}
0 & \text { if } r<a, \\
\frac{r-a}{b-a} & \text { if } a \leq r \leq b, \\
1 & \text { if } r>b
\end{array}\right.
$$

with $r \in[0,1], a=0.3$ and $b=0.8$. Once the quantifier $Q$ has been chosen, the weighting vector of the OWA operator can be obtained following Yager [25] as $w_{j}=Q(j / L)-$ $Q((j-1) / L), j=1,2, \ldots, L$. According to this, for each image $I_{i} \in \mathcal{I}$, the vector $\Theta^{i}$ obtained from $L$ subjects will be aggregated into one assessment $v^{i}$ as follows:

$$
v^{i}=w_{1} \hat{o}_{1}^{i}+w_{2} \hat{o}_{2}^{i}+\ldots+w_{L} \hat{o}_{L}^{i}
$$

where $\left[\hat{o}_{1}^{i}, \ldots, \hat{o}_{L}^{i}\right]$ is a vector obtained by ranking in nonincreasing order the values of the vector $\Theta^{i}$.

\section{FITTING THE MEMBERSHIP FUNCTION}

At this point, the aim is to obtain, for a given subset of measures $\mathcal{F} \subseteq \mathcal{P}$, the corresponding membership function $\mathcal{T}_{\mathcal{F}}$ defined as

$$
\mathcal{T}_{\mathcal{F}}: \mathbb{R}^{K^{\prime}} \rightarrow[0,1]
$$

with $K^{\prime}$ being the cardinality of $\mathcal{F}$. Since we are searching for a function which associates the measure vectors $\left(\mathbf{M}_{w}^{i}\right)$ and the human assessments of fineness $\left(v^{i}\right)$, we propose to estimate $\mathcal{T}_{\mathcal{F}}$ by fitting a suitable curve to the multiset of points $\Psi_{\mathcal{F}}=\left\{\left(\mathbf{M}_{w}^{i}, v^{i}\right), i=1, \ldots, N ; w=1, \ldots, W\right\}$, with $N$ being the number of images and $W$ the number of windows considered for each image. In this paper, for each image $I_{i} \in \mathcal{I}, W=2000$ subimages of size $32 \times 32$ have been considered (so 16000 points have been used for the fitting).

The measure values can be affected by some factors, like brightness, contrast or noise, which typically causes outliers in the fitting points. For this reason, in our approach the membership function is calculated by means of a Robust Fitting of the multiset $\Psi_{\mathcal{F}}$. In addition, the fitting could be done according to some constraints (for example, to obtain a monotonic function).

In this paper, the robust fitting based on M-estimators is used. M-estimators are a generalization of the traditional maximum likelihood estimation and, therefore, of the least squares fitting. If we consider a function $\mathcal{T}_{\mathcal{F}}$ defined by $D$ parameters $p_{1}, \ldots, p_{D}$, these parameters will be obtained as those that minimize the error function, i.e., as:

$$
\underset{p_{1}, \ldots, p_{D}}{\operatorname{argmin}} \sum_{i=1}^{N} \rho\left(\mathbf{r}_{w}^{i}\right)
$$

where $\mathbf{r}_{w}^{i}$ are the fitting residuals defined as

$$
\mathbf{r}_{w}^{i}=v^{i}-\mathcal{T}_{\mathcal{F}}\left(\mathbf{M}_{w}^{i} ; p_{1} \ldots p_{D}\right)
$$

and the function $\rho$ gives the contribution of each residual in the model [3].

It should be noticed that there is an error related to the fitting, calculated as the mean of the absolute difference between the data points and the curve. This error can also be viewed as a goodness measurement of the measures used in $\mathcal{F}$. This allows us to analyze different subsets of measures, selecting as the best subset the one with the lowest error.

For defining $\mathcal{F}$, in this paper we have focused our analysis on the cases of $K^{\prime}=1$ and $K^{\prime}=2$. The unidimensional case $\left(K^{\prime}=1\right)$ will allow to study each measure separately, comparing its goodness respect to the others. In the bidimensional case $\left(K^{\prime}=2\right)$, we will combine two measures in order to improve the individual ones (all the combinations of two elements have been considered). For higher dimensions ( $K^{\prime} \geq 3$ ), the fitting based on M-estimators is too complex, so new methods for finding $\mathcal{T}_{\mathcal{F}}$ need to be considered (for example, some kind of heuristic approaches). The case of $K^{\prime} \geq 3$ will be considered in future works. 
TABLE I

FITTING ERRORS RELATED TO EACH MEASURE AND PARAMETER VALUES FOR THE TWO MEASURES WITH LEAST ERROR

\begin{tabular}{|c|c|c|c|}
\hline Measure & Error & \multirow{2}{*}{\multicolumn{2}{|c|}{$\begin{array}{c}\text { Parameters for } \\
\text { Correlation }\end{array}$}} \\
\hline Correlation [9] & 0.1466 & & \\
\hline Amadasun [2] & 0.1515 & $a_{3}$ & -1.83828 \\
\hline Abbadeni [1] & 0.1880 & $a_{2}$ & 1.23377 \\
\hline Fractal dim. (FD) [15] & 0.1891 & $a_{1}$ & -1.20788 \\
\hline Tamura [22] & 0.1994 & $a_{0}$ & 1.03395 \\
\hline Edge density (ED) [4] & 0.2044 & $\alpha$ & 0.76851 \\
\hline DGD [11] & 0.2120 & $\beta$ & 0.02893 \\
\hline Local Homogeneity [9] & 0.2156 & & \\
\hline Short Run Emphasis [7] & 0.2211 & \multirow{2}{*}{\multicolumn{2}{|c|}{$\begin{array}{c}\text { Parameters for } \\
\text { Amadasun }\end{array}$}} \\
\hline SNE [21] & 0.2365 & & \\
\hline Weszka [24] & 0.2383 & $a_{3}$ & -0.00006 \\
\hline Newsam [14] & WSD & $a_{2}$ & 0.00556 \\
\hline Entropy [9] & WSD & $a_{1}$ & -0.17053 \\
\hline Uniformity[9] & WSD & $a_{0}$ & 1.57472 \\
\hline FMPS [26] & WSD & $\alpha$ & 16.5285 \\
\hline Variance[9] & NR & $\beta$ & 3.82800 \\
\hline Contrast [9] & NR & & \\
\hline
\end{tabular}

The unidimensional and bidimensional fittings are analyzed in the next subsections. In both cases, the following considerations will be taked into account:

1) $\mathcal{T}_{F}$ should be a monotonic function

2) The values $\mathcal{T}_{\mathcal{F}}(x)=0$ and $\mathcal{T}_{\mathcal{F}}(x)=1$ should be achieved from a certain value

\section{A. Unidimensional fitting}

Regarding the above properties, in the case of $K^{\prime}=1$ we propose to define $\mathcal{T}_{\mathcal{F}}$ as a function

$$
\mathcal{T}_{\mathcal{F}}: \mathbb{R} \rightarrow[0,1]
$$

of the form ${ }^{2}$

$\mathcal{T}_{\mathcal{F}}\left(x ; a_{n} \ldots a_{0}, \alpha, \beta\right)= \begin{cases}0 & x<\alpha, \\ \operatorname{poly}^{n}\left(x ; a_{n} \ldots a_{0}\right) & \alpha \leq x \leq \beta, \\ 1 & x>\beta\end{cases}$

with $\operatorname{poly}^{n}\left(x ; a_{n} \ldots a_{0}\right)$ being a polynomial function

$$
\operatorname{poly}^{n}\left(x ; a_{n} \ldots a_{0}\right)=a_{n} x^{n}+\ldots+a_{1} x^{1}+a_{0}
$$

In our proposal, the parameters $a_{n} \ldots a_{0}, \alpha$ and $\beta$ of the function $\mathcal{T}_{\mathcal{F}}$ are calculated by carrying out a Robust Fitting on $\Psi_{\mathcal{F}}$. For the polynomial function, the cases of $n=1,2,3$ (i.e. linear, quadratic and cubic functions) have been considered.

Table I shows, for each measure $F_{k} \in \mathcal{F}$, the least error obtained in the fitting process (sorted in increasing order of the errors). The parameter values of the two measures with the lowest error (Correlation and Amadasun) are also shown in Table I. It should be noticed that we haven't carried out the fitting with six of the measures. Four of them (marked with WSD) are rejected because their values are affected by the window size, ie., they are window size dependent. The other two (marked with NR) produce a diffuse cloud of points

\footnotetext{
${ }^{2}$ Note that this function is defined for measures that increase according to the perception of fineness. For those that decreases, the function needs to be changed appropriately
}

TABLE II

FITTING ERRORS RELATED TO EACH PAIR OF MEASURES AND

\begin{tabular}{|c|c|c|c|c|}
\hline Measure $\mathrm{x}$ & Measure y & Error & \multirow{2}{*}{\multicolumn{2}{|c|}{$\begin{array}{l}\text { Parameters for } \\
\text { FD+Amadasun }\end{array}$}} \\
\hline FD & Amadasun & 0.1095 & & \\
\hline Correlation & ED & 0.1332 & $a_{9}$ & 1.11686 \\
\hline Correlation & Tamura & 0.1339 & $a_{8}$ & -0.00076 \\
\hline Amadasun & Correlation & 0.1354 & $a_{7}$ & 0.00000 \\
\hline Abbadeni & Tamura & 0.1373 & $a_{6}$ & -0.00853 \\
\hline Amadasun & ED & 0.1431 & $a_{5}$ & -11.3885 \\
\hline Correlation & Abbadeni & 0.1433 & $a_{4}$ & 0.06165 \\
\hline Abbadeni & Amadasun & 0.1444 & $a_{3}$ & 0.22887 \\
\hline Correlation & FD & 0.1455 & $a_{2}$ & 36.8053 \\
\hline Abbadeni & FD & 0.1475 & $a_{1}$ & -1.26158 \\
\hline Tamura & Amadasun & 0.1529 & $a_{0}$ & -34.7570 \\
\hline FD & Tamura & 0.1634 & & \\
\hline ED & Tamura & 0.1636 & & \\
\hline ED & FD & 0.1764 & & \\
\hline Abbadeni & ED & 0.1799 & & \\
\hline
\end{tabular}
PARAMETER VALUES FOR THE PAIR WITH LEAST ERROR

$\Psi_{F}$ which implies that these measures are not providing a representative information about the perception of fineness.

\section{B. Bidimensional fitting}

In the case of $K^{\prime}=2$, we define $\mathcal{T}_{\mathcal{F}}$ as a function

$$
\mathcal{T}_{\mathcal{F}}: \mathbb{R}^{2} \rightarrow[0,1]
$$

of the form

$$
\begin{gathered}
\mathcal{T}_{\mathcal{F}}\left(x, y ; a_{(n !+n)} \ldots a_{0}\right)=\mathcal{T}_{\mathcal{F}}(x, y ; \text { coef })= \\
= \begin{cases}0 & p o l y 2^{n}(x, y ; \text { coef })<0, \\
p o l y 2^{n}(x, y ; \text { coef }) & 0<p o l y 2^{n}(x, y ; \text { coef })<1, \\
1 & \text { poly } 2^{n}(x, y ; \text { coef })>1\end{cases}
\end{gathered}
$$

with $\operatorname{poly} 2^{n}(x, y$; coe $f)$ being a polynomial function of two variables

$$
\begin{aligned}
& \operatorname{poly} 2^{n}\left(x, y ; a_{(n !+n)} \ldots a_{0}\right)=\sum_{i=0}^{n} \sum_{j=0}^{i} a_{(i !+j)} x^{j} y^{i-j}= \\
& =a_{(n !+n)} x^{n}+a_{(n !+n-1)} x^{n-1} y^{1}+\ldots+a_{2} x^{1}+a_{1} y^{1}+a_{0}
\end{aligned}
$$

As in the unidimensioanl case, the parameters $a_{(n !+n)} \ldots a_{0}$ of the function $\mathcal{T}_{\mathcal{F}}$ are calculated by carrying out a Robust Fitting on $\Psi_{\mathcal{F}}$. For the polynomial function, the cases of $n=1,2,3$ have been considered.

Table II shows the least fitting error obtained for each pair of measures (only the pairs compound by the first six measures in Table I are shown). It can be noticed that the use of bidimensional functions reduces the error and provides better fineness models than the unidimensional ones. In addition, the combination of two measures improves the results obtained for each measure separately. In our experiments, the pair $\mathcal{F}=\{F D$, Amadasun $\}$ gives the best results, providing the best fuzzy set for modelling the fineness. The parameter values corresponding to this member function are shown in Table II. 

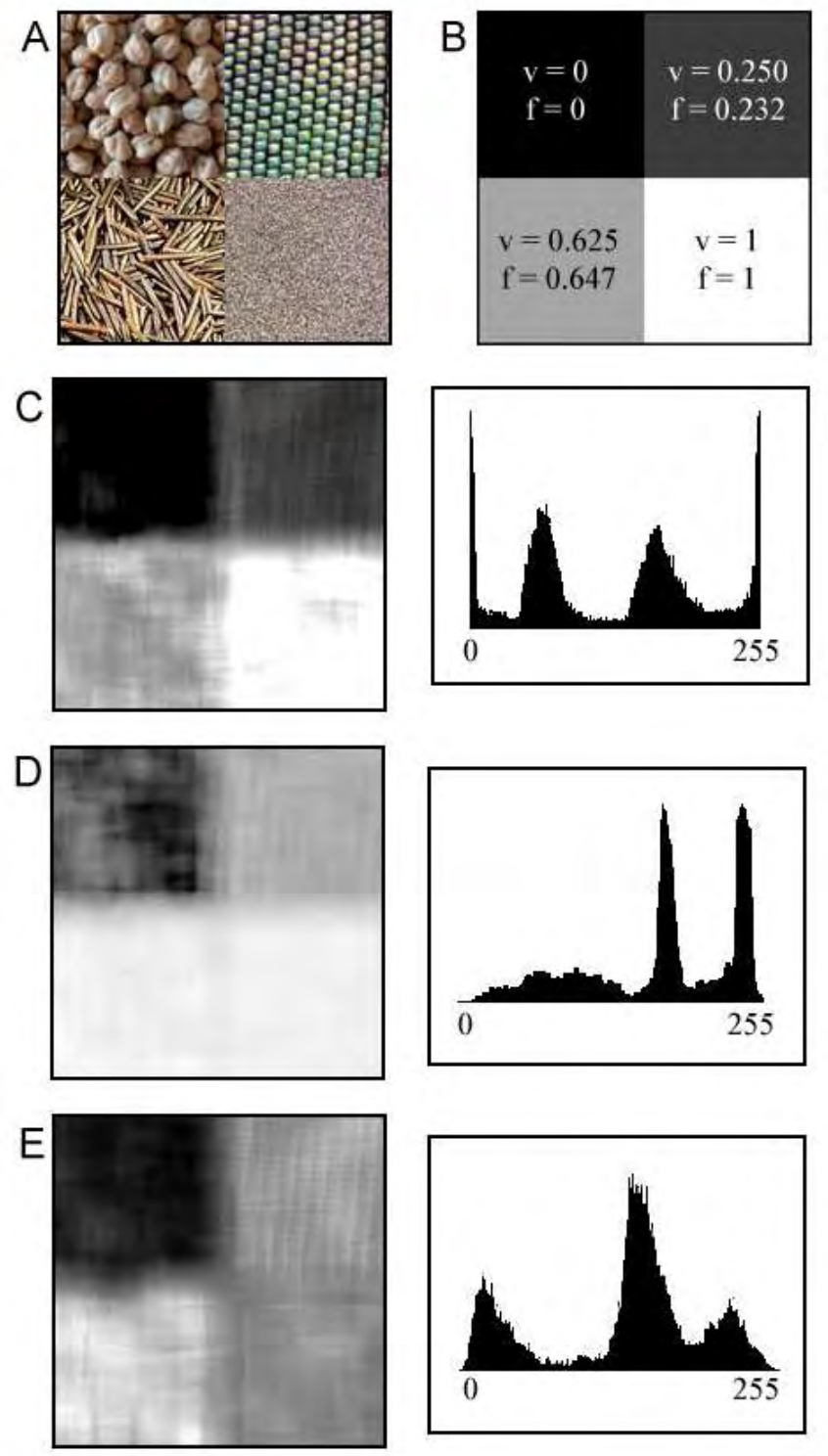

Fig. 2. Results for a mosaic image. (A) Original mosaic image (B) Degree of fineness obtained with the proposed model for the pair of measures FD+Amadasun (C)(D)(E) Fineness membership degree of each pixel obtained with the proposed model and with the individual measures Amadasun and FD, respectively, and histograms of these images

\section{RESUlTS}

In this section, the fuzzy set $\mathcal{T}_{\mathcal{F}}$ with the least fitting error, corresponding to the pair of measures $\mathcal{F}=$ $\{F D, A m a d a s u n\}$ and defined by the parameter values shown in Table II, will be applied in order to analyze the performance of the proposed model.

Let's consider Figure 2(A) corresponding to a mosaic made by several images, each one with a different increasing perception degree of fineness. The perception degree of fineness for each subimage has been calculated using the proposed model and the results are shown in Figure 2(B) where a white grey level means maximum perception of fineness, while a black one corresponds to no perception of fineness. The numeric values are also shown on each subimage: $v$ is the value of the human assessment and $f$ is the computed degree. It can be noticed that our model captures the evolution of the perception degrees of fineness.

Figure 2(C) shows a mapping from the original image to its fineness membership degree using the proposed model. For each pixel in the original image, a centered window of size $32 \times 32$ has been analyzed and its fineness membership degree has been calculated. Thus, Figure 2(C) represents the degree in which the human perceives the texture, with a white grey level meaning maximum perception of fineness, and a black one meaning no perception of fineness (i.e., maximum perception of coarseness). The histogram of this image is shown beside it. We can see four clearly differentiated peaks, corresponding to the four different fineness degrees in the image.

Figures 2(D) and 2(E) show a mapping from the original image using the Amadasun and FD measures directly (i.e., our fuzzy sets are not used). In this case, the grey level of each pixel represents the measure value, showing that these images don't provide as much perceptual information as Figure 2(C). Furthermore, their histograms don't show the four peaks corresponding to the four classes of coarseness represented in the image.

Figure 3 presents an example where the proposed fuzzy sets have been employed for pattern recognition. In this case, the figure shows a microscopy image (Figure 3(A)) corresponding to the microstructure of a metal sample [19]. The lamellae indicates islands of eutectic, which are to be separated from the uniform light regions. The brightness values in regions of the original image are not distinct, so texture information is needed for extracting the uniform areas. This fact is showed in Figure 3(B1,B2), where a thersholding on the original image is displayed (homogeneous regions cannot be separated from the textured ones as they "share" brightness values).

Figure 3(C1) shows a mapping from the original image to its "fineness" membership degree. In this case, we use a window of size $20 \times 20$. Thus, Figure $3(\mathrm{C} 1)$ represents the degree in which the human perceives the texture and it can be noticed that uniform regions correspond to areas with low degrees of fineness (i.e., high coarseness), so if only the pixels with fineness degree lower than 0.1 are selected (which it is equivalent to a coarseness degree upper than 0.9), the uniform light regions emerge with ease (Figure 3(C2,C3)).

\section{CONCLUSIONS}

In this paper, fuzzy sets for fineness perception have been defined on the domain of certain measures. The membership function associated to the fuzzy set has been learnt by finding the functional relationship between a certain measure (automatically computed over the image) and the presence degree of the textural concept. The measures have been chosen so that they collect information of the textural fineness under study whereas the presence degree has been obtained by performing a poll with humans and aggregating their assessments by means of OWA operators. Satisfactory 

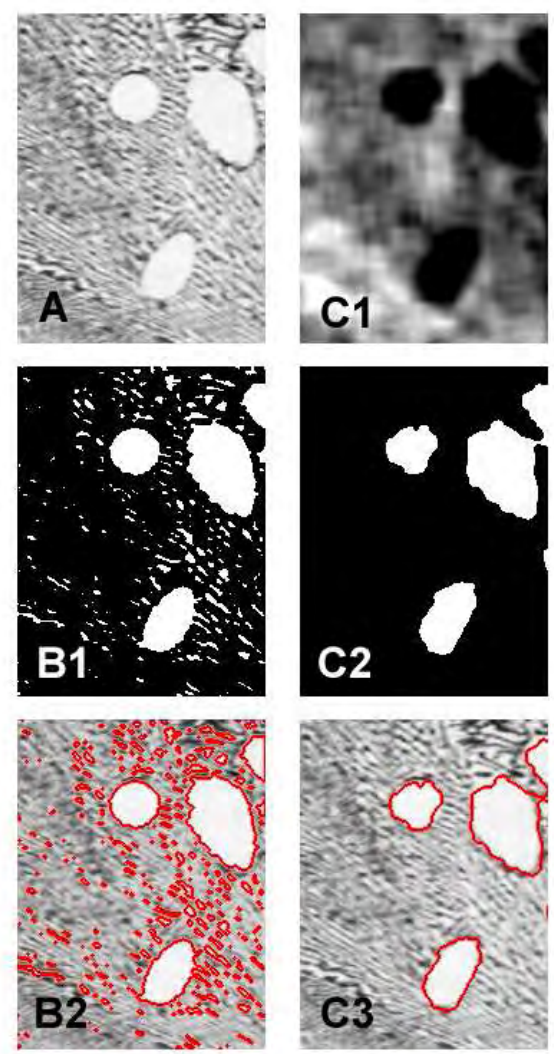

Fig. 3. Example of pattern recognition using the proposed model (A) Original image (B1) Binary image obtained by thresholding the original image (B2) Region outlines of B1 superimposed on original image (C1) Fineness membership degrees obtained with our model from the original image (C2) Binary image obtained by thresholding C1 (C3) Region outlines of $\mathrm{C} 2$ superimposed on original image

performance has been found when using the different fuzzy sets with real images and the results show a high level of connection with the assessments given by subjects.

\section{REFERENCES}

[1] N. Abbadeni, N. Ziou, and D.S. Wang. Autocovariance-based perceptual textural features corresponding to human visual perception. In Proc. of 15th International Conference on Pattern Recognition, volume 3, pages 901-904, 2000.

[2] M. Amadasun and R. King. Textural features corresponding to textural properties. IEEE Transactions on Systems, Man and Cybernetics, 19(5):1264-1274, 1989.

[3] A.E. Beaton and J.W. Tukey. The fitting of power series, meaning polynomials, illustrated on band-spectroscopic data. Technometrics, 16:147-185, 1974

[4] J. Canny. A computational approach to edge detection. IEEE Transactions on Pattern Analysis and Machine Intelligence, 8(6):679698, 1986.

[5] K.I. Chang, K.W. Bowyer, and M. Sivagurunath. Evaluation of texture segmentation algorithms. In Proc. IEEE Computer Society Conference on Computer Vision and Pattern Recognition, volume 57, pages 294 299, 2003.

[6] X. Dai and J. Maeda. Unsupervised segmentation of textured color images using fuzzy homogeneity decision. In Proc. 4th International Symposium on Video/Image Processing and Multimedia Communications, pages 75-78, 2002

[7] M.M. Galloway. Texture analysis using gray level run lengths. Computer Graphics and Image Processing, 4:172-179, 1975.
[8] M. Hanmandlu, V. K. Madasu, and S. Vasikarla. A fuzzy approach to texture segmentation. In Proc. International Conference on Information Technology: Coding and Computing, volume 1, pages 636-642, 2004.

[9] R.M. Haralick. Statistical and structural approaches to texture. Proceedings IEEE, 67(5):786-804, 1979.

[10] G. Karmakar, L. Dooley, and M. Murshed. Fuzzy rule for image segmentation incorporating texture features. In Proc. of 2002 International Conference on Image Processing, volume 1, pages I-797-I-800, 22-25 Sept. 2002.

[11] S.I. Kim, K.C. Choi, and D.S. Lee. Texture classification using run difference matrix. In Proc. of IEEE 1991 Ultrasonics Symposium, volume 2, pages 1097-1100, December 1991.

[12] S. Kulkarni and B. Verma. Fuzzy logic based texture queries for cbir. In Proc. 5th International Conference on Computational Intelligence and Multimedia Applications, pages 223-228, 2003.

[13] H.C. Lin, C.Y. Chiu, and S.N. Yang. Finding textures by textual descriptions, visual examples, and relevance feedbacks. Pattern Recognition Letters, 24(14):2255-2267, 2003.

[14] S.D. Newsam and C. Kammath. Retrieval using texture features in high resolution multi-spectral satellite imagery. In Data Mining and Knowledge Discovery: Theory, Tools, and Technology VI, SPIE Defense and Security, April 2004.

[15] S. Peleg, J. Naor, R. Hartley, and D. Avnir. Multiple resolution texture analysis and classification. IEEE Transactions on Pattern Analysis and Machine Intelligence, (4):518-523, 1984.

[16] M. Potrebić. Iterative fuzzy rule base technique for image segmentation. In Proc. of 7 th seminar on Neural Network Applications in Elecrical Engineering, pages 221-224, 23-25 Sept. 2004.

[17] A.R. Rao and G.L. Lohse. Identifying high level features of texture perception. Graphical Models and Image Processing, 55(3):218-233, 1993.

[18] Todd R. Reed and J.M. Hans Du Buf. A review of recent texture segmentation and feature extraction techniques. CVGIP: Image Understanding, 57(3):359-372, 1993.

[19] J.C. Russ. The Image Processing Handbook. CRC Press and IEEE Press, third edition, 1999.

[20] A. Shackelford. A hierachical fuzzy classification approach for highresolution multispectral data over urban areas. IEEE Transactions on Geoscience and Remote Sensing, 41(9):1920-1932, 2003.

[21] C. Sun and W.G. Wee. Neighboring gray level dependence matrix for texture classification. Computer Vision, Graphics and Image Processing, 23:341-352, 1983.

[22] H. Tamura, S. Mori, and T. Yamawaki. Textural features corresponding to visual perception. IEEE Trans. on Systems, Man and Cybernetics, 8:460-473, 1978.

[23] C.B. Wang, H.B. Wang, and Q.B. Mei. Texture segmentation based on an adaptively fuzzy clustering neural network. In Proc. of 2004 International Conference on Machine Learning and Cybernetics, volume 2, pages 1173-1176, 2004.

[24] J.S. Weszka, C.R. Dyer, and A. Rosenfeld. A comparative study of texture measures for terrain classification. IEEE Trans. on SMC, 6:269-285, 1976.

[25] R.R. Yager. On ordered weighted averaging aggregation operators in multicriteria decisionmaking. IEEE Trans. on SMC, 18(1):183-190, 1988.

[26] H. Yoshida, D.D. Casalino, B. Keserci, A. Coskun, O. Ozturk, and A. Savranlar. Wavelet-packet-based texture analysis for differentiation between benign and malignant liver tumours in ultrasound images. Physics in Medicine and Biology, 48:3735-3753, 2003. 